\title{
Pulsed Accretion in the T Tauri Binary TWA 3A
}

\author{
Benjamin M. Tofflemire ${ }^{1}$, Robert D. Mathieu ${ }^{1}$, Gregory J. Herczeg ${ }^{2}$, Rachel L. Akeson ${ }^{3}$, and David R. Ciardi ${ }^{3}$ \\ ${ }^{1}$ Department of Astronomy, University of Wisconsin-Madison, 475 North Charter Street, Madison, WI 53706, USA \\ ${ }^{2}$ The Kavli Institute for Astronomy and Astrophysics, Peking University, Beijing 100871, China \\ ${ }^{3}$ NASA Exoplanet Science Institute, IPAC/Caltech, Pasadena, CA 91125, USA \\ Received 2017 May 15; accepted 2017 May 30; published 2017 June 14
}

\begin{abstract}
TWA 3A is the most recent addition to a small group of young binary systems that both actively accrete from a circumbinary disk and have spectroscopic orbital solutions. As such, it provides a unique opportunity to test binary accretion theory in a well-constrained setting. To examine TWA 3A's time-variable accretion behavior, we have conducted a two-year, optical photometric monitoring campaign, obtaining dense orbital phase coverage $(\sim 20$ observations per orbit) for $\sim 15$ orbital periods. From $U$-band measurements we derive the time-dependent binary mass accretion rate, finding bursts of accretion near each periastron passage. On average, these enhanced accretion events evolve over orbital phases 0.85 to 1.05 , reaching their peak at periastron. The specific accretion rate increases above the quiescent value by a factor of $\sim 4$ on average but the peak can be as high as an order of magnitude in a given orbit. The phase dependence and amplitude of TWA 3A accretion is in good agreement with numerical simulations of binary accretion with similar orbital parameters. In these simulations, periastron accretion bursts are fueled by periodic streams of material from the circumbinary disk that are driven by the binary orbit. We find that TWA 3A's average accretion behavior is remarkably similar to DQ Tau, another T Tauri binary with similar orbital parameters, but with significantly less variability from orbit to orbit. This is only the second clear case of orbital-phase-dependent accretion in a T Tauri binary.
\end{abstract}

Key words: accretion, accretion disks - binaries: close - stars: formation - stars: individual (TWA 3A)

\section{Introduction}

TWA 3, also known as Hen 3-600, is a pre-main-sequence (pre-MS) star system composed of two spatially resolved components: TWA 3A, a spectroscopic binary hosting a circumbinary accretion disk, and TWA 3B, a diskless tertiary at a separation of $1 . " 5$ ( $\sim 52.5 \mathrm{au}$ in projection; de la Reza et al. 1989; Jayawardhana et al. 1999; Andrews et al. 2010; Kellogg et al. 2017). This multi-star architecture offers a unique opportunity to investigate the impact multiplicity has on the distribution and evolution of circumstellar material during star formation. In this Letter, we focus on the spectroscopic binary, TWA 3A, monitoring its accretion behavior in order to characterize accretion flows in the binary environment. Table 1 presents the relevant binary and disk characteristics for TWA 3A.

Binary and higher-order multiple systems are observed as a frequent outcome of star formation (Raghavan et al. 2010; Kraus et al. 2011). Our understanding of binary population statistics has advanced with large-scale imaging and spectroscopic surveys, yet the impact binarity has on the star-disk interaction and planet formation remains poorly understood. The ubiquity of binaries, along with the growing number of planets found in, and around, binary systems (Orosz et al. 2012; Kaib et al. 2013; Mugrauer et al. 2014), motivates a detailed characterization of the binary-disk interaction.

Close binaries deviate most from the single-star paradigm where orbital dynamics are capable of sculpting the distribution and flows of disk material. For systems with semimajor axes less than $\sim 100$ au, orbital resonances are capable of dynamically clearing a region of disk material around the binary, opening the possibility for three stable disks: a circumstellar disk around each star and an encompassing circumbinary disk (Artymowicz \& Lubow 1994). Theory predicts that rather than completely damming the inflow of material from the circumbinary disk, accretion will proceed in discrete, periodic streams that form at the inner edge of the circumbinary disk. These streams cross the cleared gap supplying mass to small circumstellar disks or accreting directly onto the stars themselves (Artymowicz \& Lubow 1996; Günther \& Kley 2002; Muñoz \& Lai 2016).

The frequency of these streams and their impact on the stellar mass accretion rate are predicted to be highly dependent on the binary orbital parameters. Focusing on eccentric, equalmass binaries, similar to TWA 3A, numerical simulations predict that every apastron passage $(\phi=0.5)$ will induce a stream of circumbinary material that leads to an accretion event near periastron passage $(\phi=0 ; 1)$. These episodes are predicted to increase the specific accretion rate by up to a factor of 10. This periodic accretion behavior has been observed in the T Tauri binary DQ Tau (Mathieu et al. 1997; Tofflemire et al. 2017).

The accretion streams predicted by the binary-disk interaction are likely important astrophysical phenomena at a variety of scales. From giant planet formation spurring streams across disk gaps (Lubow \& D'Angelo 2006) to accretion onto binary black holes (Bowen et al. 2017), the same physical processes are at play. Interferometry and adaptive optics techniques are beginning to spatially resolve such structures in pre-MS systems (Beck et al. 2012; Casassus et al. 2013; Yang et al. 2017), but they are unable to describe their temporal characteristics. Accretion in short-period, pre-MS binaries offers a unique regime to probe the dynamics of accretion streams.

\section{Observations and Data Reduction}

In order to characterize the accretion behavior of TWA $3 \mathrm{~A}$, we have conducted a long-term, moderate-cadence, optical 
Table 1

TWA 3A System Characteristics

\begin{tabular}{lcc}
\hline \hline Parameter & Value & References \\
\hline $\mathrm{P}($ days $)$ & $34.87846 \pm 0.00090$ & $(1)$ \\
$\gamma\left(\mathrm{km} \mathrm{s}^{-1}\right)$ & $+10.17 \pm 0.40$ & $(1)$ \\
$e$ & $0.6280 \pm 0.0060$ & $(1)$ \\
$\mathrm{T}_{\text {peri }}(\mathrm{HJD}-2,400,000)$ & $52704.554 \pm 0.063$ & $(1)$ \\
$a\left(R_{\odot}\right)$ & $46.51 \pm 0.49$ & $(1)$ \\
$q \equiv M_{2} / M_{1}$ & $0.841 \pm 0.014$ & $(1)$ \\
$M_{1}\left(M_{\odot}\right)$ & $0.6027 \pm 0.0207$ & $(1)$ \\
$M_{2}\left(M_{\odot}\right)$ & $0.5072 \pm 0.0158$ & $(1)$ \\
Periastron Separation $\left(R_{\odot}\right)$ & $17.30 \pm 0.33$ & $(1)$ \\
Apastron Separation $\left(R_{\odot}\right)$ & $75.72 \pm 0.85$ & $(2)$ \\
$i_{\text {disk }}\left({ }^{\circ}\right)$ & 36 & $(2)$ \\
Disk $M_{\text {dust }}\left(M_{\odot}\right)$ & $7 \times 10^{-6}$ & $(3)$ \\
$v$ sin $i\left(\mathrm{~km} \mathrm{~s}^{-1}\right)$ & 20 & $(4)^{\mathrm{a}}$ \\
$\mathrm{d}(\mathrm{pc})$ & $30 \pm 3$ & $(5)$ \\
$A_{V}$ & $0.04 \pm 0.3$ & \\
\hline
\end{tabular}

Note. (1) Kellogg et al. (2017; assuming $i_{\text {binary }}=i_{\text {disk }}=36^{\circ}$ ), (2) Andrews et al. (2010), (3) Torres et al. (2003), (4) Ducourant et al. (2014), (5) This work.

${ }^{a}$ Kinematic distance derived using the Ducourant et al. (2014) position, proper motion, and convergent point with the Kellogg et al. (2017) $\gamma$ velocity.

photometric monitoring campaign using the Las Cumbres Observatory (LCO) and SMARTS queue-scheduled facilities.

\subsection{LCO $1 \mathrm{~m}$ Network}

The LCO $1 \mathrm{~m}$ network comprises nine $1 \mathrm{~m}$ telescopes located across four global sites: Siding Springs Observatory (Australia), SAAO (South Africa), CTIO (Chile), and McDonald Observatory (USA). Spanning $\sim 12$ TWA $3 \mathrm{~A}$ orbital periods, our observations were made between 2014 May and 2016 April. Observing visits were scheduled 20 times per orbit while the target was visible (airmass $<2$ ), corresponding to a cadence of $42 \mathrm{hr}$. Each visit consisted of three images in the $U B V R$ filters. All data are reduced by the LCO pipeline applying bad-pixel, bias, dark, and flat-field corrections. The three images per filter per visit are than aligned, combined, and fit with astrometric solutions using standard IRAF tasks.

\subsection{SMARTS $1.3 \mathrm{~m}$}

The SMARTS $1.3 \mathrm{~m}$ telescope at CTIO is outfitted with the ANDICAM detector. Our program requested every-other-night visits of TWA 3A while it was visible (airmass $<2$ ) between 2014 December and 2016 July. Each visit consisted of three images in the $B$ and $V$ filters. Data are reduced with the SMARTS pipeline, which applies bias and flat-field corrections. Each set of images per visit are aligned, combined, and fit with an astrometric solution using standard IRAF tasks.

\subsection{Photometry and Calibration}

For each telescope (LCO; SMARTS) and filter set (UBVR; $B V)$, SExtractor (Bertin \& Arnouts 1996) is used to perform automated source detection and photometry on each image producing time-series instrumental magnitudes. A source catalog for each data set is then created from spatial matching of the astrometric solutions. Due to poor seeing and/or telescope focus, flux from TWA 3A and TWA 3B could not be consistently separated. As such, SExtractor parameters were optimized to photometer the entire TWA 3 system.
Each source catalog is then fed into an ensemble photometry routine following the Honeycutt (1992) formalism. By selecting non-varying comparison stars interactively, variations from airmass and nightly observing conditions are corrected, resulting in relative-magnitude light curves for each star in the field of view (FOV). We note that this correction does not include color information, which leaves some small systematic error, especially in the $U$-band where atmospheric corrections are most color dependent.

Relative ensemble magnitudes are then transformed to apparent magnitudes using non-varying stars in the LCO FOV for which published empirical or derived photometry exists. Five such stars are present in the LCO FOV (TYC 7213-797-1, 7213-391-1, 7213-1239-1, 7213-933-1, and 7213-829-1). Their $V$ magnitudes range from 11.18 to 11.57 and $(B-V)$ colors span 0.71 to 2.00 . Using empirical measurements where available, we draw $B$ and $V$ values from the All-Sky Compiled Catalogue (Kharchenko 2001). For $R$-band, we use a colorless transformation from the Carlsberg Meridian Catalog 15 (CMC15; Niels Bohr Institute et al. 2014) $r^{\prime}$ to $R\left(R=r^{\prime}-0.22 \pm 0.12\right)$ derived from 3690 overlapping stars between CMC15 and the Lowell Observatory Near-Earth Object Search (Skiff 2007). Without empirical $U$-band measurements available, we use the fitted apparent $U$-band magnitudes derived by Pickles \& Depagne (2010). From these five stars we compute zero-point and color transformations that are applied to the rest of the field. Systematic errors associated with this calibration procedure are determined from the root-mean-squared deviation between the transformed published values of the calibrating stars in color-magnitude space. They are $0.18,0.22$, 0.25 , and $0.07 \mathrm{mag}$ for $U, B, V$, and $R$ bands, respectively. (Systematic errors are propagated through the mass accretion rate derivation that follows and are presented in Figure 3.)

Not all five of the calibration stars in the LCO FOV are present in the smaller $6^{\prime} \mathrm{FOV}$ of the ANDICAM CCD. To transform the SMAR TS data to apparent magnitudes, we bootstrap the apparent magnitudes derived for non-varying stars in the LCO FOV that overlap with the SMARTS FOV and use those to determine zero-point and color transformations.

\section{Analysis \\ 3.1. Light Curve Variability}

Figure 1 presents the $U_{-}, B-, V$-, and $R$-band light curves for TWA 3 plotted against an arbitrary orbital cycle number set to 1 for the first observed periastron passage. In each panel, vertical dashed lines mark the TWA 3A periastron passage and horizontal dotted lines mark the quiescent value (average of orbital phases 0.2 to 0.4 ). Brightening events near periastron passages are seen consistently, having the largest increase in the $U$-band. These events very closely match the accretion behavior predicted for eccentric binaries.

To ensure variability observed in the TWA 3 system is indeed from the disk-bearing binary and not the tertiary, we perform point-spread-function photometry on a subset of the SMARTS $B$-band images where the light from each component can be reliably separated. Figure 2 displays the result where TWA $3 \mathrm{~A}$ is the clear source of variability. The standard deviation of these light curves are 0.14 and 0.02 mag for TWA 3A and TWA 3B, respectively. In the following, we assume all variability in the TWA 3 system results from the spectroscopic binary.

Before assigning all optical variability to changes in the TWA 3A accretion rate, we inspect our light curves for 


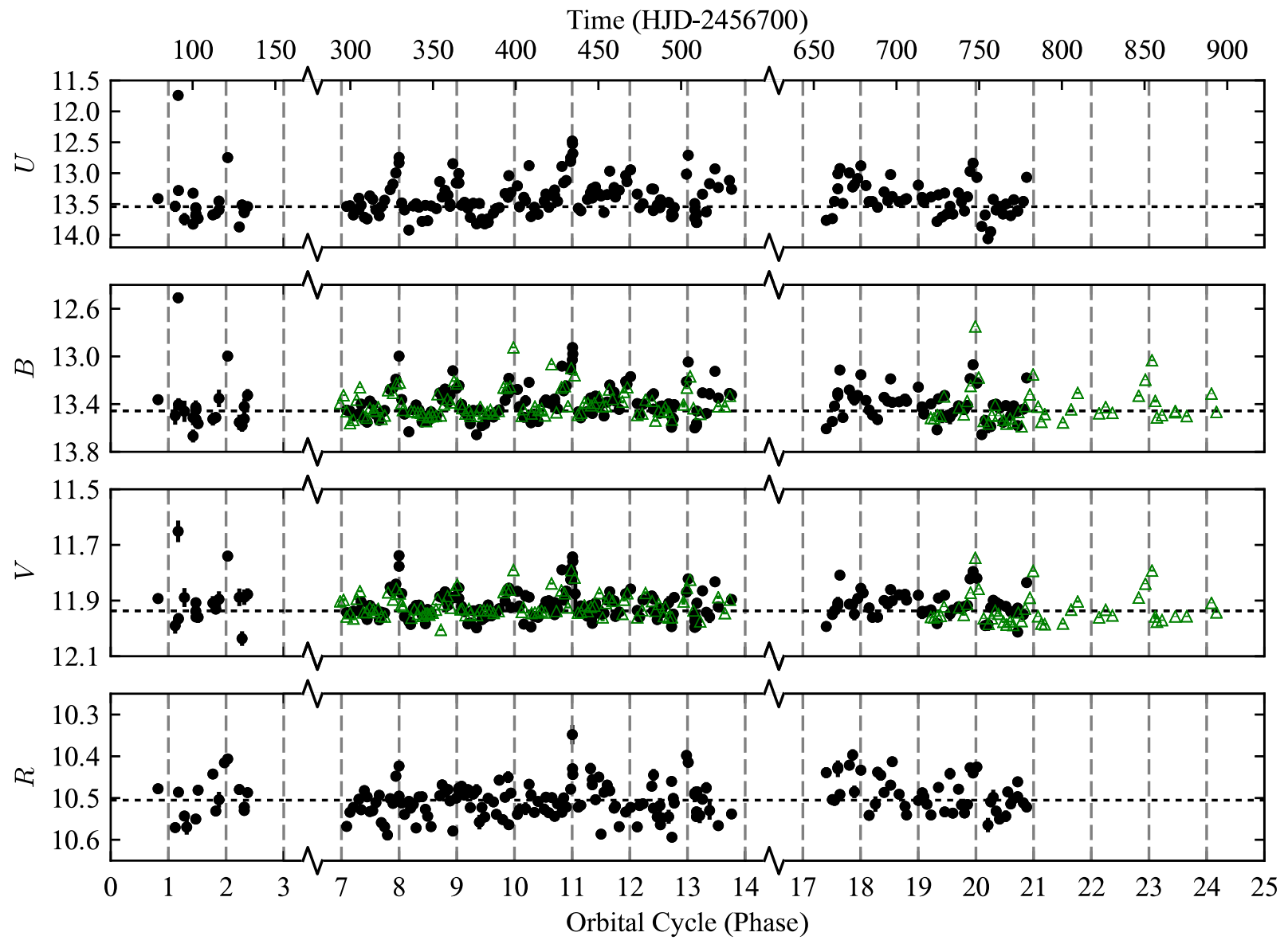

Figure 1. TWA 3 UBVR light curves plotted against arbitrary orbital cycle number. The corresponding heliocentric Julian date (HJD) is presented on the top y-axis. LCO and SMARTS data are represented as circles and triangles, respectively. Vertical dashed lines mark periastron passages. Horizontal dashed lines mark the quiescent flux level.

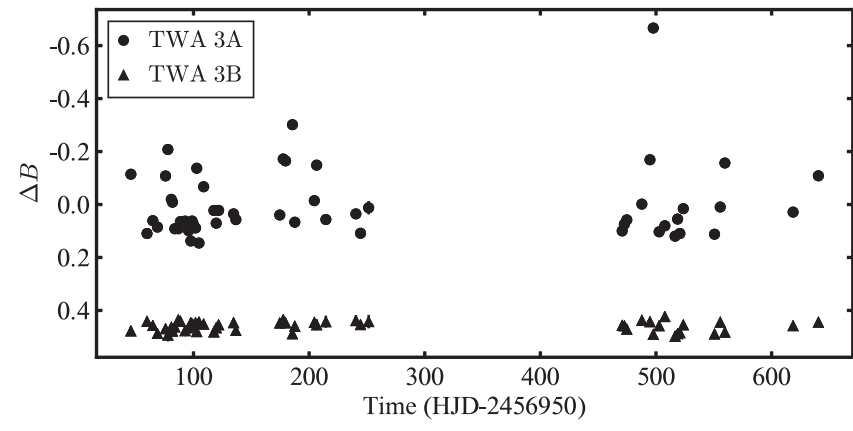

Figure 2. SMARTS $B$-band light curve of TWA 3 A (circles) and TWA 3B (triangles) from observations in which point-spread-function photometry can separate the contribution from both components. The TWA 3A quiescent magnitude is $\sim 0.38$ mag brighter than TWA $3 \mathrm{~B}$.

contributions from stellar flares (magnetic reconnection events at stellar surfaces). In high-cadence photometry of DQ Tau, stellar flares with amplitudes greater than $\Delta U=0.32 \mathrm{mag}$ were found to have a temporal contribution of $\sim 3 \%$ (Tofflemire et al. 2017). Assuming the same contribution in TWA 3A corresponds to six measurements. In moderatecadence photometry, however, these events would likely go undetected having similar amplitudes and colors as accretion variability. Only if a measurement were to contain a large flare or a flare peak, where the photometric color is typically bluer than accretion, would it stand out from the underlying accretion variability (Kowalski et al. 2016).
One measurement in our light curves has a color, magnitude, and temporal behavior that suggest it contains a stellar flare. Occurring at orbital cycle $\sim 1.2$ in Figure 1, it is the brightest $U$-band measurement by $0.7 \mathrm{mag}$ and the bluest in $(U-B)$ by $0.2 \mathrm{mag}$, well separated from both observed distributions. The associated flux would correspond to a factor of $\sim 15$ increase in the mass accretion rate compared to other measurements at similar orbital phases and a factor of two greater than the next highest measurement. A measurement only $5 \mathrm{hr}$ later, however, falls securely within the remaining spread for that orbital phase. Accretion events of this scale are expected to be rare and to evolve over much longer timescales (e.g., Cody et al. 2014). And critically, the three $U$-band images combined in this measurement show a rapid $\sim 0.2$ mag decline over $\sim 3$ minutes. Given these characteristics, we conclude this measurement contains a stellar flare and remove it. In the following analysis, we assume the remaining variability is due to changes in the TWA 3A accretion rate.

\subsection{Mass Accretion Rate}

Flux-calibrated $U$-band photometry can be used to derive a mass accretion rate with knowledge of the distance and extinction to the source, the photospheric $U$-band flux in the absence of accretion, and the stellar mass and radius. With values for the distance and stellar parameters, we determine the extinction and photospheric properties following Herczeg \& Hillenbrand (2014). First, we fit a spatially resolved Keck LRIS spectrum of TWA 3A (Herczeg et al. 2009) with a library of 
empirical weak-lined T Tauri star (WTTS) spectra. The spectra are fit with three free parameters: a flux normalization, an additive accretion spectrum, and the extinction. Our results are consistent with those in Herczeg \& Hillenbrand (2014), namely, $A_{V}=0.04( \pm 0.30 \mathrm{mag})$ and a combined TWA 3A spectral type of M4.1 ( \pm 0.3 subclasses). Second, the bestfitting WTTS spectrum is convolved with a $U$-band filter to determine the underlying photospheric contribution of the binary.

Because our $U$-band measurements are for the entire TWA 3 system, we use a spatially resolved Keck LRIS spectrum of TWA 3B (Herczeg et al. 2009) to determine its $U$-band contribution. Assuming a distance of $30 \mathrm{pc}$, we extinction correct the $U$-band measurements and convert them to $U$-band luminosities. Subtracting the contribution from TWA 3B and the underlying TWA $3 \mathrm{~A}$ photosphere, we arrive at the TWA 3A $U$-band accretion luminosity. Using the model-dependent, empirical relation derived in Gullbring et al. (1998), we calculate the total accretion luminosity from the $U$-band as follows:

$$
\log \left(L_{\text {Acc }} / L_{\odot}\right)=1.09 \log \left(L_{U_{\text {excess }}} / L_{\odot}\right)+0.98 .
$$

For a single star, the mass accretion rate can be determined from the accretion luminosity with the following:

$$
\dot{M} \simeq \frac{L_{\mathrm{Acc}} R_{\star}}{G M_{\star}}\left(1-\frac{R_{\star}}{R_{\text {in }}}\right)^{-1},
$$

where $R_{\text {in }}$ is the magnetospheric disk truncation radius from which material free falls along magnetic field lines (typically assumed to be $5 R_{\star}$; e.g., Johnstone et al. 2014).

Our measurements, however, are of the combined accretion luminosity from two stars with different masses and radii. Without a theoretical consensus for which star should predominantly receive the mass from circumbinary accretion flows, we assume that each star accretes at the same rate. The accretion luminosity emitted from the primary star alone, $L_{\mathrm{Acc}, 1}$, becomes

$$
L_{\mathrm{Acc}, 1}=L_{\mathrm{Acc}, \text { Total }}\left(1+q \frac{R_{\star, 1}}{R_{\star, 2}}\right)^{-1}
$$

where $q$ is the mass ratio. The total mass accretion rate for the binary is then

$$
\dot{M} \simeq 2 \frac{L_{\mathrm{Acc}, 1} R_{\star, 1}}{G M_{\star, 1}}\left(1-\frac{R_{\star, 1}}{R_{\text {in }}}\right)^{-1} .
$$

For the stellar radii, we use the Dotter et al. (2008) stellar evolution models to compute the average radii for 0.6 and 0.5 $M_{\odot}$ stars between 5 and $10 \mathrm{Myr}$. They are 1.06 and $0.99 R_{\odot}$, respectively. $R_{\text {in }}$ is set to the canonical single-star value of $5 R_{\star}$. The derived accretion rates range between $0.8 \times 10^{-11}$ and $2.4 \times 10^{-10} M_{\odot} \mathrm{yr}^{-1}$, in good agreement with previous measurements (Muzerolle et al. 2000; Herczeg et al. 2009). Since the stars have near equal masses, the choice to split the accretion rate equally between the two corresponds to only a $\pm \sim 5 \%$ difference from assigning all the accretion to one star.

The top panel of Figure 3 presents the mass accretion rate phase-folded about the orbital period. The repeated enhanced accretion events observed near periastron increase the accretion rate by a factor of $\sim 3-10$ from the quiescent value.
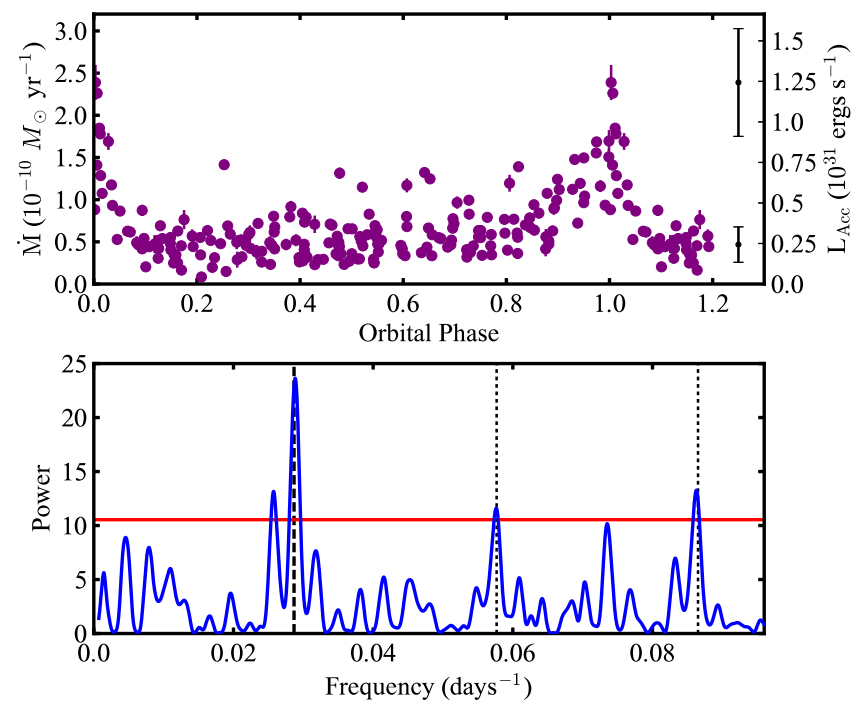

Figure 3. Top: TWA $3 \mathrm{~A}$ mass accretion rate and accretion luminosity phasefolded about the orbital period. Black error bars on the right correspond to the propagation of the systematic error of our photometric calibration. Because the systematic error is relative, we present it for the quiescent and peak accretion rate values. Bottom: periodogram of the mass accretion rate measurements. Significant power is found above the $99 \%$ false-alarm probability (horizontal red line) consistent with the orbital period (vertical dashed line).

\subsection{Accretion Periodicity}

To determine the significance of the periodic accretion behavior, we perform a Lomb-Scargle periodogram (Scargle 1982) on the accretion rate measurements. The bottom panel of Figure 3 presents the power spectrum. A significant peak is observed above the $99 \%$ false-alarm probability with a period of $34.67 \pm 0.14$ days, in good agreement $(1.5 \sigma)$ with the binary orbital period. (Error in the accretion rate period is derived from a $10^{6}$ iteration Monte Carlo bootstrap simulation using random sampling with replacement of the $\dot{M}$ and HJD measurement pairs; Press et al. 1992.) Additional higherfrequency peaks occurring at two and three times the peak frequency result from the varying and non-sinusoidal morphology of the enhanced accretion events. The small peak to the left of the primary does not remain significant after filtering the data at the primary frequency.

\subsection{Accretion Rate Profile}

The morphology of enhanced accretion events contains information on the interaction between the binary orbit and the circumbinary mass flows. In order to compare our observations with numerical simulations, we create an average accretion rate profile as a function of orbital phase. Breaking the orbitalphase-folded data into bins of $\phi=0.05$ (our sampling rate), we calculate the median mass accretion rate for each bin and set the bin error as its standard deviation. The result is presented in the top panel of Figure 4 where, on average, the accretion rate is elevated between orbital phases 0.85 and 1.05 , reaching a peak near periastron of $\sim 4$ times the average quiescent value.

In the bottom panel of Figure 4, we compare the TWA 3A accretion rate profile to a simulation of binary accretion (Muñoz \& Lai 2016) and to the DQ Tau accretion rate profile (Tofflemire et al. 2017). Both have been normalized to the TWA 3A average mass accretion rate. The Muñoz \& Lai (2016) simulation shown is a 2D hydrodynamical model using the adaptive-meshrefinement code AREPO (Springel 2010) for an equal-mass 

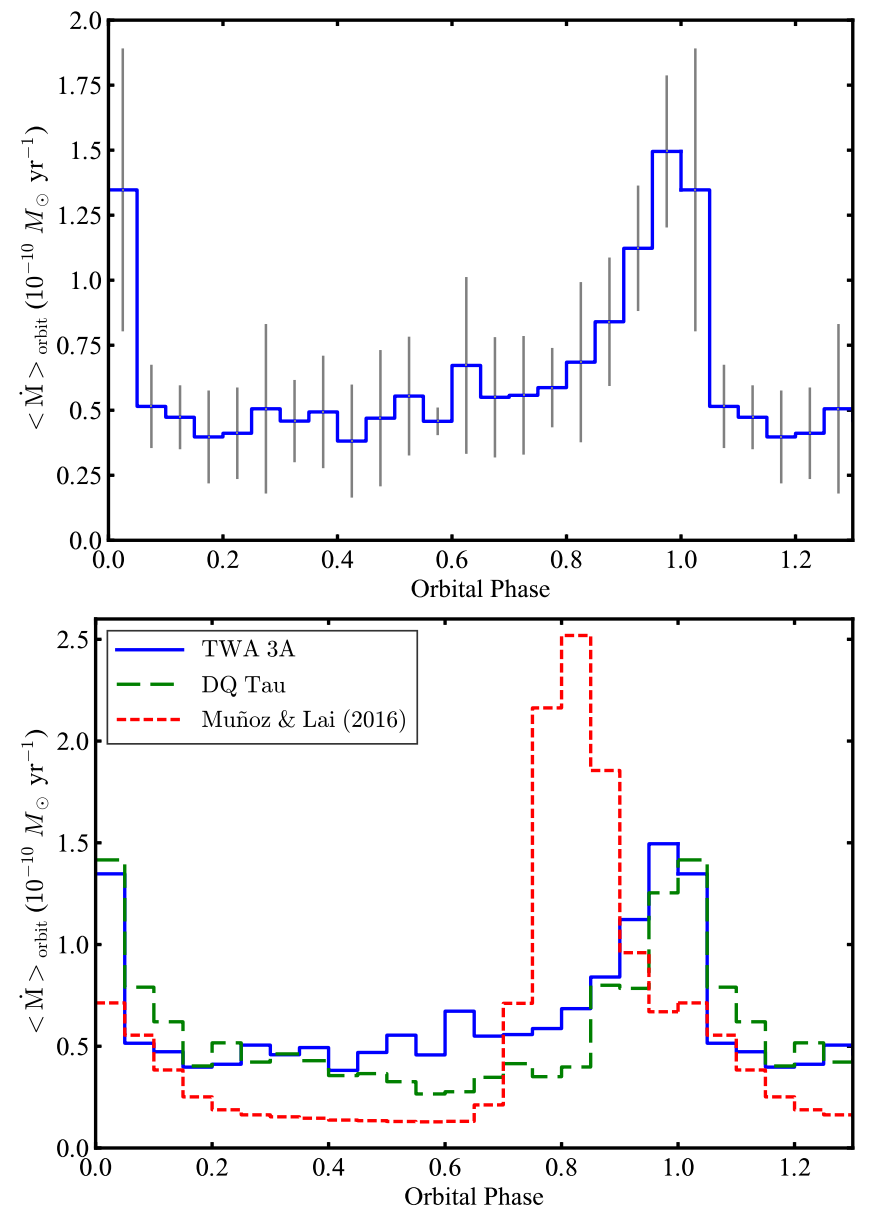

Figure 4. Top: TWA $3 \mathrm{~A}$ median accretion rate as a function of orbital phase. Error bars represent the standard deviation within each phase bin. Bottom: comparison of the TWA 3A accretion rate profile with DQ Tau (Tofflemire et al. 2017) and a numerical simulation from Muñoz \& Lai (2016) for a binary with similar orbital parameters.

binary with an eccentricity of 0.5 . Ten consecutive orbital periods of the simulation were used to create the accretion profile.

\section{Discussion}

Figures 3 and 4 provide conclusive evidence that accretion in TWA 3A is strongly influenced by the binary orbit, leading to periodic accretion bursts near periastron passage. This behavior is largely consistent with the prediction of numerical simulations supporting the scenario that periodic streams of mass are capable of carrying material across a cleared gap to the central binary. Similar behavior has only been this clearly observed in one other binary, DQ Tau. The binary UZ Tau E (Jensen et al. 2007) and the protostar LRLL 54361 (Muzerolle et al. 2013; unknown period) are also intriguing sources that have shown hints of phase-dependent accretion.

Direct comparisons to numerical simulations can begin to constrain the dynamics of accretion streams. Despite a notable phase offset, there is good agreement between TWA 3A and the Muñoz \& Lai (2016) model. Making more in-depth comparisons, however, is not straightforward. The difficulty lies in the relevant hydrodynamic and magnetic scales that effect stable circumstellar disk material in short-period systems.

In high-resolution hydrodynamic simulations, each star develops a stable circumstellar disk that collects and organizes incoming circumbinary stream material. Accretion events in this case result from a combination of tidal torques that each star induces on its companion's disk and the interaction of the circumbinary streams with the circumstellar disks. Without magnetic fields, material is accreted once it reaches the stellar surfaces. These stable circumstellar disks have the effect of regularizing accretion events from orbit to orbit.

In short-period systems, however, there is a close match between the outer dynamical truncation radii of circumstellar disks imposed by binary orbital resonances and the inner magnetic truncation radii typically assumed for single stars. If magnetic fields are capable of disrupting or reducing the size of stable circumstellar disks, there may be a direct interaction of stream material with the stellar magnetosphere that is not captured by current models.

We note that in lower-resolution simulations by Günther \& Kley (2002), each star develops a marginally resolved circumstellar disk, yet their accretion rate profile peaks directly at periastron, matching the observations of TWA 3A and DQ Tau. It is unclear whether resolved circumstellar disks or different disk treatments (viscosity, radiative cooling, viscous heating, etc.) are responsible for these differences in the timing and amplitude of accretion events. Short of including magnetic fields in three dimensions, a study varying the disk properties and inner accretion radii would provide a more suitable comparison with the data presented here.

Finally, the TWA 3A and DQ Tau average accretion rate profiles are striking similar. Although they have similar orbital parameters, DQ Tau exhibits much more variability from orbit to orbit (compare Figure 3 to 7 in Tofflemire et al. 2017). Yet, on average, their profiles are very similar in shape and amplitude. Regularity in TWA 3A accretion events when compared to DQ Tau, may be the result of a lower overall accretion rate $\left(\sim 10^{-2}\right)$, or perhaps its larger semimajor axis, permits some amount of stable circumstellar disk material that regularizes accretion events.

\section{Summary}

With a long-term, densely sampled, optical photometric monitoring campaign, we have characterized the accretion behavior of the young binary TWA 3A. Here, we summarize the main results of our work:

1. Spatially resolved photometry reveals that accretion variability from the spectroscopic binary, TWA 3A, is the dominant source of optical variability in the combined light of the TWA 3 system.

2. From $U$-band observations we derive the TWA 3A mass accretion rate as a function of time. Periodic accretion events are observed near each periastron passage. On average, the accretion rate is elevated between orbital phases 0.85 to 1.05 , reaching a peak of $\sim 4$ times the quiescent value.

3. The observed behavior is in good agreement with numerical simulations, providing strong evidence for periodic circumbinary accretion streams in TWA 3A. This is only the second clear case of orbital-phasedependent accretion in a pre-MS binary.

4. These are some of the first data that can begin to constrain the dynamical properties of accretion flows. Comparisons with current models are limited, however, as they do not include the disruptive effect magnetic fields have on stable circumstellar disks. 
5. The TWA $3 \mathrm{~A}$ average accretion rate profile is remarkably similar to that of DQ Tau (a shorter-period system with similar mass ratio and eccentricity; Tofflemire et al. 2017) despite DQ Tau's larger variability from orbit to orbit.

The authors would like to thank Lisa Prato for providing the TWA 3A spectroscopic orbital solution prior to its publication. This work makes use of observations from the LCO network and the CTIO $1.3 \mathrm{~m}$ telescope operated by the SMARTS Consortium. This research has made use of the VizieR catalogue access tool, CDS, Strasbourg, France. B.T. acknowledges funding from Sigma Xi Honors Society and from the University of Wisconsin-Madison Graduate School.

\section{References}

Andrews, S. M., Czekala, I., Wilner, D. J., et al. 2010, ApJ, 710, 462 Artymowicz, P., \& Lubow, S. H. 1994, ApJ, 421, 651

Artymowicz, P., \& Lubow, S. H. 1996, ApJL, 467, L77

Beck, T. L., Bary, J. S., Dutrey, A., et al. 2012, ApJ, 754, 72

Bertin, E., \& Arnouts, S. 1996, A\&AS, 117, 393

Bowen, D. B., Campanelli, M., Krolik, J. H., Mewes, V., \& Noble, S. C. 2017, ApJ, 838, 42

Casassus, S., van der Plas, G., M., S. P., et al. 2013, Natur, 493, 191

Cody, A. M., Stauffer, J., Baglin, A., et al. 2014, AJ, 147, 82

de la Reza, R., Torres, C. A. O., Quast, G., Castilho, B. V., \& Vieira, G. L. 1989, ApJL, 343, L61

Dotter, A., Chaboyer, B., Jevremović, D., et al. 2008, ApJS, 178, 89

Ducourant, C., Teixeira, R., Galli, P. A. B., et al. 2014, A\&A, 563, A121

Gullbring, E., Hartmann, L., Briceno, C., \& Calvet, N. 1998, ApJ, 492, 323

Günther, R., \& Kley, W. 2002, A\&A, 387, 550

Herczeg, G. J., Cruz, K. L., \& Hillenbrand, L. A. 2009, ApJ, 696, 1589
Herczeg, G. J., \& Hillenbrand, L. A. 2014, ApJ, 786, 97

Honeycutt, R. K. 1992, PASP, 104, 435

Jayawardhana, R., Hartmann, L., Fazio, G., et al. 1999, ApJL, 520, L41

Jensen, E. L. N., Dhital, S., Stassun, K. G., et al. 2007, AJ, 134, 241

Johnstone, C. P., Jardine, M., Gregory, S. G., Donati, J.-F., \& Hussain, G. 2014, MNRAS, 437, 3202

Kaib, N. A., Raymond, S. N., \& Duncan, M. 2013, Natur, 493, 381

Kellogg, K., Prato, L., Torres, G., et al. 2017, ApJ, submitted

Kharchenko, N. V. 2001, KFNT, 17, 409

Kowalski, A. F., Mathioudakis, M., Hawley, S. L., et al. 2016, ApJ, 820,95

Kraus, A. L., Ireland, M. J., Martinache, F., \& Hillenbrand, L. A. 2011, ApJ, 731, 8

Lubow, S. H., \& D’Angelo, G. 2006, ApJ, 641, 526

Mathieu, R. D., Stassun, K., Basri, G., et al. 1997, AJ, 113, 1841

Mugrauer, M., Ginski, C., \& Seeliger, M. 2014, MNRAS, 439, 1063

Muñoz, D. J., \& Lai, D. 2016, ApJ, 827, 43

Muzerolle, J., Calvet, N., Briceño, C., Hartmann, L., \& Hillenbrand, L. 2000, ApJL, 535, L47

Muzerolle, J., Furlan, E., Flaherty, K., Balog, Z., \& Gutermuth, R. 2013, Natur, 493, 378

Niels Bohr Institute, University of Copenhagen, Institute of Astronomy Cambridge, \& Real Instituto y Observatorio de La Armada en San Fernando 2014, yCat, 1327, 0

Orosz, J. A., Welsh, W. F., Carter, J. A., et al. 2012, Sci, 337, 1511

Pickles, A., \& Depagne, É. 2010, PASP, 122, 1437

Press, W. H., Teukolsky, S. A., Vetterling, W. T., \& Flannery, B. P. 1992, Numerical Recipes in FORTRAN. The Art of Scientific Computing (2nd ed; Cambridge: Cambridge Univ. Press)

Raghavan, D., McAlister, H. A., Henry, T. J., et al. 2010, ApJS, 190, 1

Scargle, J. D. 1982, ApJ, 263, 835

Skiff, B. A. 2007, yCat, 2277, 0

Springel, V. 2010, MNRAS, 401, 791

Tofflemire, B. M., Mathieu, R. D., Ardila, D. R., et al. 2017, ApJ, 835, 8

Torres, G., Guenther, E. W., Marschall, L. A., et al. 2003, AJ, 125, 825

Yang, Y., Hashimoto, J., Hayashi, S. S., et al. 2017, AJ, 153, 7 